\title{
COSTAS ALICANTINAS Y COSTAS MAGREBIEES: EL ESPACIO MARÍTIMO MUSULMÁN SEGÚN LOS TEXTOS ÁRABES
}

Por

MÍKEL DE EPALZA

Alicante árabe es definida por las fuentes musulmanas como ciudad y como fortaleza (EPALZA, 1985), pero en sus relaciones exteriores se caracteriza por ser cabeza de fortalezas en la Vía Augusta (RUBIERA, 1985a) y por ser puerto y atracadero (marsà, mina $\left.\bar{a}^{\prime}\right)$. El texto fundamental de Al-Idrisi, a mediados del siglo XII, menciona especialmente sus exportaciones marítimas (de productos del esparto) y sus atarazanas para la fabricación de diversas clases de naves.

En este trabajo se va a estudiar, en una primera parte, un texto poco conocido del geógrafo onubense del siglo Xl "Ubayd Alläh Al-Bakri, sobre las costas de Sharq Al-Andalus (Oriente o Levante de la Península lbérica) en relación con sus vecinas costas del Mágreb. En una segunda parte, se traerán a colación una serie de textos árabes paralelos (Al-Yacqūbi del siglo $I X ; I b n$ Hawqal, del $X ; A l$-Idrisi, del XII; Ibn Sacid Al-Magribi, del XIII), para mejor comprender los rasgos fundamentales de este espacio marítimo. Finalmente, se harán algunas consideraciones geográficas generales, para mejor comprender el alcance de estos textos.

Excluimos la zona o espacio maritimo del Estrecho (az-zuqāq) y sus costas adyacentes, que suelen tener un tratamiento especial en las fuentes geográficas árabes - aun dentro de la conocida oposición de "las dos orillas" (al-cudwatān)- y la zona àctualmente andáluza de Almería, que tuvo siempre en la Edad Media, por su posición geográfica, una situación común a todas las costas andaluzas y a las de Sharq Al-Andalus, donde se le colocó a menudo, al menos hasta el siglo XIII.

Este texto de Ubaidállah Al-Bakri ha sido a veces utilizado por su descripción de las costas del Levante de la Península de Al-Andalus (RLBIERA, 1985b), dentro de los muchos estudios sobre las costas levantinas (LA CHICA, 1974), que recuerdan los más detallados portulanos cartográficos bajomedievales (EPALZA, 1986). Lo he utilizado yo mismo para la historia árabe de Santa Pola (SÁNCHEZ-BLASCO-GUARDIOLA, 1986, 19). Aquí lo queremos presentar como la mejor expresión, en época árabe, del espacio marítimo entre la costa levantina y la magrebí, dentro de una doble 
tradición del período árabe: la de la navegación mediterránea entre esas costas y la de los textos árabes que, durante casi cuatro siglos, se refieren en particular a esta zona marítima y su navegabilidad.

Es evidente que gracias a esos textos podemos ponderar esa "vía prioritaria hacia Oriente», que indica la importancia de este "Canal de la Mancha del Mediterráneo" (Braudel) y la función geopo'ítica de las costas de Sharq Al-Andalus como upuerta de Al-Andalus hacia Orienten (EPALZA, 1984).

\section{El texto de Ubaidállah Al-Bakri}

La edición clásica de lo que nos ha llegado de la obra de este geógrafo andalusí de fines del siglo XI se debe a Mac Guckin De Slane (Argel, 1911-1913, reimpresión 1965). Es la que vamos a seguir. Eliseo Vidal Beltrán tradujo al castellano la parte referente a España (VIDAL, 1982), pero como el texto que aquí se va a presentar estaba en la parte magrebl de la obra, no lo incluyó en su libro.

He aquí la traducción castellana del texto, con transcripción intercalada y alguna nota de identificación de algunas palabras árabes (véanse mapas 1 y 2):

"En cuanto a la relación de los puertos (marāsi, plural de marsà, "fondeadero"), a partir del puerto de Aslan hacia el este, el que está más cerca es el puerto de Al-Mā' Al-Madfün ("el agua enterrada"). Sus casas están sobre un cementerio. Tiene fuentes de agua que corren hacia el mar. Entre esos dos puertos hay trece millas. Tiene enfrente, en tierras de Al-Andalus (barr Al-Anda/us), Marsà Ar-Rāhib ("el puerto del cura, del monje"). Del uno al otro hay dos etapas (maŷrayān) y un tercio.

Le sigue a seis millas (amyâl, plural de mil) el puerto del monte de Orán (Wahrānl, puerto grande (marsà kabir, Mazalquivir), protegido de toda clase de vientos. Tiene enfrente, en tierras de Al-Andalus, el puerto antiguo de Aškübiras, donde los marinos fondeaban antes de desembarcar en Baŷyāna (Pechina, antigua capital de Almería). Del uno al otro hay dos etapas y media.

Le sigue, también hacia el este, a cuarenta millas por tierra, el puerto de 'Ain Furrüŷ ("Fuente de Pollos", actual Bethioua). Es un puerto seguro, de invierno. Tiene pozos de agua. Sus casas están sobre un cementerio. Tiene enfrente, en tierras de Al-Andalus, el puerto de Aguilas (Aqila), que es el puerto de la ciudad de Lorca (Lürqa). Del uno al otro hay tres etapas.

Le sigue, hacia el este, el puerto de Oașr Al-Fulüs ("Fortaleza de las Monedas"), que es una ciudad junto al mar, que no está habitada. Tiene agua traída artificialmente y que se saca hurgando en el suelo. Corresponde, en tierras de Al-Andalus, al puerto de Cartagena (Qartaŷanna).

Le sigue, a treinta y cinco millas, el puerto de Magỉa Bani Hāsim (fracción Maguila de los Háshim). Es un puerto de verano, que no está protegido de los vientos. Tiene una fortaleza religiosa (ribātt) a orillas del mar, con gente. Tiene mucha agua. Tiene enfrente, en tierras de Al-Andalus, a Captel de Tudmir (Qabțil Tudmir, en la parte norte del Mar Menor, según Al-Idrisi, Ilamado "de Tudmir" para diferenciarlo de su homónimo de la desembocadura del Guadalquivir).

Le sigue el puerto de la ciudad de Tenes, con los puertos de lițābi ("poco importantes", según traducción de De Slane) entre los dos. Es puerto de verano, protegido por el este y por el oeste. Tiene agua que surge a la superficie. El puerto de Tenes tiene enfrente, en tierras de Al-Andalus, Santa Pola (Sant Bül).

Sigue al puerto de Tenes, hacia el este, a más de veinte millas, el puerto de la isla de Wuaūr ("Las Cavidades"). Tiene un pequeño río que vierte sus. aguas en el 
mar. La isla está cerca de tierra firme (barr, también "la costa" como opuesta a "mar", como en la ya mencionada expresión barr Al-Andalus, "tierras, tierra firme o costa de Al-Andalus"). Tiene enfrente, en tierras de Al-Andalus, el puerto de Alicante (Laqant). Se corta el mar, entre los dos, en cinco etapas.

Después está el puerto de Cherchel, que tenía una ciudad que fue importante antiguamente y que no está habitada. Se puede sacar agua del suelo. Está protegida por el este y el oeste. La ciudad de Cherchel tenía un puerto construido (minā'), que ahora está rellenado. Hay fortalezas religiosas (ribāțāt) en las que se reúne cada año mucha gente. Tiene enfrente, a cinco jornadas y media, el puerto de Mudaira (Moraira, por mala transcripción del texto árabel.

Le sigue el monte Sanwa (Chenoua). Tiene un puerto llamado Al-Batāal. No está habitado. Está protegido de los vientos del oeste. Tiene agua que corre. Tiene enfrente. a cinco jornadas, en la orilla opuesta de Al-Andalus, el monte Qarūn (hay que leer Qawün, como Al-Idrisi, el Montgó de Denia).

Después está el puerto de Hūr. Después, hacia Anf Al-Qanātir ("Cabo de los Arcos"). Después, hacia Marsà Ad-Dubbān ("Puerto de las Moscas").

Le sigue el puerto de Yanabiya (lectura dudosa). Tiene una isla. Aquí había antiguamente una ciudad. No está habitada. Tiene un río que se echa al mar. Tiene enfrente, en las tierras de Al-Andalus, a seis jornadas, el puerto de Denia (Dāniva).

Le sigue el puerto de Argel (Al-Ŷazä'ir, "Las Islas"), conocido por Las islas de los Banu Mazganna. Ya hemos mencionado anteriormente esta ciudad. Es un puerto seguro, tanto del este como del oeste, para invernar, entre la isla Satufula y la tierra firme. Hay en el puerto una fuente de agua dulce. Tiene enfrente, en las tierras de Al-Andalus, a siete jornadas, el puerto de Peñíscola (Baniškula).

Sigue a este puerto, entre los puertos más famosos, Marsà Ad-Dâyyây ("Puerto de las Gallinas"). Es para el verano y no es seguro. Tiene enfrente, por parte de la península de Al-Andalus, la isla de Mallorca.

Después viene el puerto de la ciudad de Bujía (Bî̀āya, actualmente transcrito en Argelia Bidjaïa), antigua, poblada, bien construida, con gente de Al-Andalus. Al este, tiene un río grande (la Soumam) en el que pueden entrar las naves cargadas. Es un puerto seguro, para invernar. A partir de aquí no hay correspondencia con la Pęininsula de Al-Andalus" (DE SLANE, 1911-13, 81-82).

Es evidente que este texto se merece ya algunos comentarios, para sacarle la información que contiene y que supera ampliamente las meras afirmaciones de la traducción.

En primer lugar, se trata de la descripción de todo un espacio marítimo, entre las dos orillas, del Mágreb y Al-Andalus, abundantemente mencionadas como opuestas. Los árabes tenían una expresión acuñada: al-cudwatān, "las dos orillas", como si se tratara de las dos orillas de un río, el pasadizo de mar que separa las costas del Mágreb y de Al-Andalus, consideradas estas tierras también como dos islas o dos penínsulas, la "Isla del Al-Andalus» separada de todo lo que le rodeaba por el mar y los Pirineos, y la "Isla de Mágreb", también aislada, por el mar y el desierto del Sáhara. La oposición de las dos orillas (el texto de Al-Bakri utiliza la expresión explícitamente en la referencia Cherchel-Montgól es mucho más que una referencia geográfica y se utilizará continuamente después del siglo XI, cuando aumenten las relaciones políticas de Al-Andalus con los almorávides, almohades y Mariníes del Mágreb.

Es significativo, aunque complejo, el hecho de que esta relación entre las dos 
costas se describa sólo a partir del Mágreb y no cuando se habla de las costas de Al-Andalus.

El espacio marítimo se describe con una doble medida: las etapas o jornadas, para atravesar el mar, y las millas, para las distancias por tierra, entre los diversos puntos costeros. Es evidente que estos textos están basados no sólo en la distribución ptolemaica de los espacios sino también en la experiencia marinera de los viajeros marítimos.

En medidas decimales, la distancia entre la ciudad de Alicante y la costa magrebí más cercana es de unos $200 \mathrm{kms}$. y de la ciudad de Orán, unos 250, según cálculos que me ha facilitado amablemente el profesor José Costa Mas, de la Universidad de Alicante.

La experiencia marinera es sobre todo, en el Mágreb, de navegación de cabotaje, sin relaciones con el interior de las tierras: los datos fundamentales costeros para los autores árabes como Ubaidállah Al-Bakri son la protección y seguridad de los fondeaderos contra las tempestades y la capacidad de abastecerse en agua. Sólo se mencionan villorrios, con algún "castillo fortificado" que atraía a las gentes del interior para cumplir con los deberes de la "guerra santa" o yihäd en las costas fronteras del Islam. No hay ciudades costeras (el texto subraya repetidas veces que están deshabitadas). Sólo tienen la categoría de ciudad (madina) Bujía, Argel y Tenes.

La ciudad de Tenes adquiere en esa costa magrebí opuesta a la alicantina un relieve especial, durante toda la Edad Media: se le llama en nuestro texto una vez "ciudad" y tres más "puerto". Era realmente, como dos siglos antes en el texto de Al-Yáqūbi, el punto final del cabotaje magrebí proveniente de Oriente. Desde alli se bifurcaban las vías: por tierra, hacia el sur, hacia Tiaret y las zonas comerciantes del Mágreb medio y el Sáhara; por mar, hacia Al-Andalus, por las costas de Tudmir, ahora alicantino-murcianas; eventualmente, seguían el cabotaje hacia el Mágreb extremo y El Estrecho (Al-Magrib Al-Aqșà wa-z-Zuqāq). De esta situación estratégica de Tenes $y$ de su calidad de cruce de caminos sacaría ese puerto costero riqueza comercial y su estructura claramente urbana.

\section{Textos paralelos sobre este espacio marítimo}

La significación esencial de este texto de Ubaidállah Al-Bakri como expresión de la importancia de este espacio o via marítima queda más evidente al cotejarlo con otros textos medievales árabes, que indican todos ellos unas constantes.

El primero sería el del oriental $A /$-Yacqūbi (finales del siglo $(X)$, con el itinerario desde Cairuán, çapital de Ifriqiya o Mágreb oriental, a Córdoba, capital de Al-Andalus.

"Salga de Qairawān hacia Túnez. Allí se embarca y viaja por mar durante diez días, siguiendo la costa y sin penetrar tierra adentro, hasta que se encuentra enfrente de la Península de Al-Andalus, en un lugar llamado Tenes, que está en la costa a cuatro jornadas de la ciudad de Tahert (por aquel entonces capital de los Rustumies, en el interior de la Argelia actual o Mágreb mediol. Se dirige entonces a la Península de Al-Andalus cortando la alta mar durante un día y una noche, hasta que llega al pais de Tudmir, región amplia y habitada, en la que hay dos ciudades llamadas respectivamente Al-cAskar y Lorca, ambas con mezquita mayor. Después se sale hacia... Córdoba... (por) Elbira..." (DE GOEJE, 1892, 353-354; EPALZA, 1984, 94).

Tres elementos se pueden destacar en este texto: el punto de vista del autor, que escribe desde Oriente Medio e inicia, por tanto, su relación desde Oriente; el que señala muy claramente las dos grandes regiones que se enlazaban por ese itinerario marítimo, Ifriqiya (Mágreb oriental con su capital Cairuán, la zona más arabizada y 
orientalizada del Norte de África) y Al-Andalus, en su totalidad, con su capital Córdoba, en situación central, y sus costas de acceso directo desde Oriente, las de Tudmir o zona alicantino-murciana. Ya he analizado con más pormenor la función de "Puerta de Al-Andalus» que los árabes atribuyeron con razón a esas costas (EPALZA, 1984).

El texto de Ibn Hawqal (también oriental, de mediados del siglo $x^{\circ}$, que viajó personalmente a Al-Andalus) no sólo realiza un itinerario de este a oeste, siguiendo la costa magrebí, sino que describe las principales poblaciones costeras. Encontramos así tres referencias explícitas de relaciones del Mágreb medio con las costas de Sharq Al-Andalus (ver mapa 3).

"TABARQA. Es un pueblo, que está enfrente para la gente de Al-Andalus (' $u d$ watan li-ah/ Al-Andalusl, que allí llegan y de alli se embarcan hacia Al-Andalus... Se hizo famosa porque alli llegaban muchas naves con andalusies y comerciantes, que allí se detenían y se asociaban antiguamente. Está orientada hacia las primeras tierras de Al-Andalus (awā'il Al-Andalus), desde donde está. También está enfrente de algunos territorios de los franŷ (francos, catalanes)" (IBN HAWOAL, 1979, 76).

Este primer texto de Ibn Hawqal sitúa más al este que Al-Bakri lque empezaba en Bujía) la relación de las costas magrebíes con las andalusies, tanto del punto de vista geográfico como comercial, un elemento fundamental de su libro (Tabarca está en la actual frontera tunecino-argelina). Desde el punto de vista comercial, Ibn Hawqal se refiere a una época pasada, seguramente al siglo $\mid X^{\circ}$, antes de la ocupación de las costas del Mágreb oriental y central por los fatimíes, enemigos de los omeyas de Al-Andalus.

"TENES. Es una ciudad con murallas y con muchas puertas... Es la ciudad a donde más van los andalusíes, desde la otra orilla, con sus barcos para comerciar, y de allí van a otros sitios...".

Ibn Hawqal confirma aquí la riqueza del centro comercial que representa Tenes para los navegantes y comerciantes de Al-Andalus, y su situación de nudo de comunicaciones (norte-sur, este-oeste), en la costa magrebí frente al Sharq Al-Andalus, como ya lo advirtiera Al-Yacubi, medio siglo antes.

"ORÁN... Hay allí negociantes hábiles. Es puerto (farda) de Al-Andalus."

Esta zona específica, la de Orán, será señalada como centro de exportaciones hacia Al-Andalus, dos siglos después, por Al-ldrisi.

En efecto, este autor de mediados del XII ( $y$, por tanto, posterior a Ubaidállah Al-Bakri, al que debe muchas informaciones) también habla de relaciones de las dos costas, a partir de la costa magrebí, como Al-Bakri.

"ORÁN... Está enfrente de la ciudad de Almería, en la costa marítima de AlAndalu's. La anchura del mar entre las dos es de dos jornadas. De allí sobre todo se proveen las costas de Al-Andalus de cereales" (PETTI SUMA, 1972, 252).

La palabra árabe mira ("aprovisionamiento de grano») indica una de las riquezas constantes de las llanuras oranesas, frente al déficit cerealístico endémico de la Península.

Acabaremos la serie de textos paralelos al de Al-Bakri con dos del siglo XIII, del antólogo literario y geógrafo Ibn $S a^{c} i d$ Al-Magribi. En su obra geográfica nos describe la ciudad de Tenes y la relaciona - es una constante de las costas del Mágreb medio - con la costa de Al-Andalus (AL-ARABI, 1970, 142). 
"La ciudad de Tenes es muy conocida por su abundancia de trigo. De alli se lo llevan con barcos a las costas de Al-Andalus y a otros sitios...

Desde Mostaganem, la anchura del mar hasta la costa de Denia, en Al-Andalus, es aproximadamente de tres jornadas y media (maȳāri, también "etapas", "jornadas")...

... Y la anchura del mar por Bujía (Bî̀āya) en dirección a Tortosa, en Al-Andalus, es de tres jornadas".

El texto de Ibn Saíd, aparte de un error de distancia (pone tres jornadas de Bujía a Tortosa, donde Al-Bakri había puesto, más de acuerdo con la realidad y los otros textos árabes, siete desde Argel a Peñíscola), confirma el límite septentrional de la costa levantina de Al-Andalus: Peñíscola-Tortosa. A partir de ahí, se considera que el "Canal de la Mancha del Mediterráneo" sólo tiene, frente a las costas magrebies, las islas Baleares y Cerdeña.

Ibn Saíd Al-Mágribi, en su antología literaria, obra de varias generaciones de antólogos, agrupa a sus literarios por su lugar de nacimiento, que describe con unas frases tópicas. Al hablar de Alicante, confirma la naturaleza de este espacio marítimo. descrito casi cuatro siglos antes por Al-Yacubi.

"ALICANTE. Tiene un territorio ('amal) grande, especializado en la producción de higos secos y aceite. Sus vinos son proverbiales (madkūra), excelentes (mufadụala) y famosos por su fuerza (mašhüra bi-l-quwwa). Esta ciudad tiene puerto fondeadero (mina') para los barcos. Es el puerto atracadero (marsà) de Murcia, donde se embarca la gente para Ifriqiya. Tiene una fortaleza (qal al que parece una punta de lanza hacia el cielo; no he visto en todo Al-Andalus una fortaleza mejor fortificada (amnacu min-hā) » (DAYF, II, 274).

Esta referencia a Alicante "puerto de Murcia» (en Al-Yacubi se mencionaba no la capital de la región, sino todo Tudmir) para ir a Ifriqiya (en vez de mencionar su vieja capital Cairuán, en plena decadencia frente a Túnez, en el siglo XIII) muestra algunas de las variantes políticas entre el siglo $I X^{\circ} \mathrm{V}$ el $X I I^{\circ}$. Pero indica un mismo espacio vial marítimo, entre el Oriente de Al-Andalus y el Mágreb oriental, por el constante camino marítimo árabe que unía a Al-Andalus con Oriente por el espacio marítimo que unía las costas de Sharq Al-Andalus con las del Mágreb medio.

\section{Conclusiones}

El geógrafo enciclopedista sirio del XIII Yãqūt Al-Hamawi veía muy bien desde Oriente, según la tradición árabe y mencionando expresamente a Ibn Hawqal, ese extremo o final occidental del Mediterráneo o Mar de Siria (al-bahr ašsămi). Lo veía evidentemente como un canal entre dos orillas: "El mercader de Mosul Ibn Hawqal, que había recorrido muchos países escribiendo lo que veía, dice... La tierra de AlAndalus confronta por el mar con tierras del Mágreb, partiendo de Túnez y pasando por Tabarca, Yazā'ir Banū Mazgannay, Ankür (Nakur), Ceuta y finalmente Azilā y mar océano" (ABD AL-KARIM, 1974, 91-92).

Pero esta visión no era puramente cartográfica. Representa un itinerario de navegación por el mar y una afinidad entre las dos orillas, por las particulares condiciones favorables de esta zona marítima.

En primer lugar, está la cercanía geográfica entre las dos costas. Además, pertenecían ambas, durante todo el período que va desde el siglo $V I I I^{\circ}$ al XIII ${ }^{\circ}$, a un mismo mundo árabe islámico (a pesar de períodos de enfrentamiento político entre soberanos musulmanes, como el omeya-aglabi del VIII-IX o el omeya-fatimi del X). Pero sobre todo es un espacio marítimo bonancible, generalmente alejado de los vientos y corrien- 
tes atlánticos de la zona del Estrecho y de las tempestades del golfo de León, al norte de las Baleares.

Por eso fue zona de acceso a la Península, ya en la antigüedad pre-islámica, de los cartagineses de Cartago a Cartagena y de los bizantinos del Bizanceno lahora Sahel tunecino) al Levante de la Península, en el siglo $V 1^{\circ}$. Este "canal» fue también aprovechado por la colonización francesa en Argelia (Conquista de 1830 desde Menorca; emigraciones españolas al Oranesado en el siglo XIX; hasta vuelos aéreos primitivos desde Francia, atravesando el Mediterráneo desde el aeropuerto alicantino de El Altet; etc.).

Con la Independencia argelina de 1962, este mar asume la condición política de frontera entre el Mágreb árabe y España. Pero esta frontera puede no ser ideológicamente enfrentada (Norte-Sur), sino complementaria y permeable, como lo era en la Edad Media árabe.

ABD AL-KARIM, 1974: G. 'ABD AL-KARIM, La España musulmana en la obra de Yâqût (s. XII-XIII), Granada DE GOEJE, 1892: M. J. DE GOEJE ed., Al-Yacqưbî. Kitâb al buldân, Leiden, 2." ed.

DE SLANE, 1965: MAC GUCKIN DE SLANE, Description de I'Afrique septentrionale par Abou-Obeäd-El-Bekri, Argel, 19111913, reimpr.

EPALZA, 1984: M. de EPALZA, "Los beréberes y la arabización del País Valencianon, Miscel.tània Sanchis Guarner, Ouaderns de Filologia Valenciana, Valencia, $1,9 \mathrm{i}-100$.

EPALZA, 1985: "Estudio del texto de Al-Idrisi sobre Alicanten, Sharg Al-Andalus. Estudios Árabes, 2.

EPALZA, 1986: "El portulano de Ibrahim de Murcia", Sharq Al-Andalus. Estudios Árabes, 3.

IBN HAWOAL, 1979: IBN AWOAL, Kitâb şûrat al-arọ, Beirut.

LA CHICA, 1974: M. LA CHICA, "Referencias árabes a las costas de la Peninsula lbérica en la Edad Media", Saitabi, Valencia, XXIV, $55-63$

PETTI SUMA, 1972: M. T. PETTI SUMA y otros, Al-ldrîsî. Opus Geograficum, fasc. lli, Nápoles-homa.

RUBIERA, 1985a: M. J. RUBIERA, Villena en las calzadas romana $y$ árabe, Alicante.

RUBIERA, 1985b: M. J. RUBIERA, La Taifa de Denia, Alicante.

SÁNCHEZ-BLASCO-GUARDIOLA, 1986: M. J. SANCHEZ - E. BLASCO - A. GUARDIOLA, Portus IIIicitanus. Datos para una sintesis, Santa Pola.

VIDAL, 1982: E. VIDAL BELTRAN, Abũ CUbayd Al-Bakrí. Geogratia de España (Kitâb al-masâlik wa-l-mamâlik), Zaragoza. 